\title{
Type Ia Progenitor Hunt in Ancient Remnants
}

\author{
Wolfgang E. Kerzendorf \\ Research School of Astronomy and Astrophysics, Mount Stromlo Observatory, Cotter Road, \\ Weston Creek, ACT 2611, Australia \\ email: wkerzend@mso .anu.edu.au
}

\begin{abstract}
There is broad agreement that the stars which explode as Type Ia supernovae are white dwarfs. They have accreted material in a binary system until they are near the Chandrasekhar mass and detonate/deflagrate. The two main scenarios for this accretion process are merging with a companion white dwarf (double degenerate scenario), or accretion from a mainsequence to red giant donor (single degenerate scenario). The donor star survives post-explosion and would provide substantial evidence for the single degenerate scenario, if found. Our team is analyzing stars in close proximity to Galactic Type Ia remnants to find surviving donor stars. In my talk I will introduce the different progenitor systems and the expected state for a donor star today. I will outline our search using high resolution spectroscopy and will present updated results.
\end{abstract}

Keywords. stars: abundances, supernovae — supernova remnants

\section{Introduction}

Type Ia supernovae (SNe Ia) are important and powerful tools in modern astronomy. They have a major impact on planet formation through nucleosynthesis, stellar astrophysics, and the chemical evolution of the Universe. Due to their relative homogeneity they also serve as powerful cosmological probes. It is therefore unfortunate that the progenitors of these explosions and the explosion physics are still poorly understood.

The community agrees that these explosions are powered by the deflagration/ detonation of carbon/oxygen white dwarfs close to the Chandrasekhar mass $\left(1.38 M_{\odot}\right)$. Most stars however leave white dwarfs with $0.6 M_{\odot}$ and no star leaves a remnant as heavy as $1.38 M_{\odot}$, which implies that white dwarfs need to acquire mass if they are to explode as SNe Ia. Currently there are two main scenarios for this mass acquisition. In the single degenerate scenario the white dwarf accretes matter from a normal, nondegenerate companion star (donor star). An important aspect of this scenario is that the companion to the white dwarf should survive the explosion and be visible afterwards. In the second scenario, the double degenerate scenario, two white dwarfs merge (with a total combined mass of more than $\left.1.38 M_{\odot}\right)$ and explode. This scenario does not predict a surviving companion. Despite concerted effort over the past 20 years, the primary question remains: Which binary scenario can explain the observed SNe Ia explosions?

In the case of the single degenerate there is a very strong and testable prediction: the surviving donor star. This paper will outline the search for such a donor star performed by our group and will mention other efforts where applicable. In Section 2 we will outline the expected state of the donor post-explosion and will highlight potential features which allow for easy identification. We will present the searchable remnants in Section 3 and will describe our efforts in finding a donor star in some of those. We will conclude in Section 4 with a discussion of our findings and will outline future work. 


\section{The Donor Star Post-Explosion}

We will highlight three possible features of a donor star post explosion: unusual kinematics, unusual rotation, and an unusual state.

After explosion, the donor gets flung from the site with the previous orbital velocity. Detailed binary population synthesis calculations (Han 2008) have found the escape velocity for a wide range of donor stars. Main-sequence donors have the highest velocities with up to $200 \mathrm{~km} \mathrm{~s}^{-1}$, subgiants have escape velocities of roughly $150 \mathrm{~km} \mathrm{~s}^{-1}$ and giants have the lowest velocities with roughly $80 \mathrm{kms}^{-1}$. In addition, the supernova ejecta impart a kick to the donor star, which is normally much smaller than the orbital velocities (Canal et al. 2001). The excess velocity is measured as two components: radial velocity and proper motion. As only the radial velocity component can be measured with high accuracy, there are cases where the splitting into radial velocity and proper motion combined with the kinematic noise of the Galaxy hides the unusual spatial motion of a donor.

During the Roche Lobe Overflow phase, the donor star will tidally couple and will inherit post-explosion the orbital frequency of the system as stellar rotation. Kerzendorf et al. (2009) suggest that this stellar rotation could be a distinguishing feature for donor stars. Using again the results of Han (2008), the main-sequence donor will have a very high rotation velocities of up to $150 \mathrm{~km} \mathrm{~s}^{-1}$, the subgiant will have rotational velocities around $100 \mathrm{~km} \mathrm{~s}^{-1}$, and a giant donor will have rotational velocities around $60 \mathrm{~km} \mathrm{~s}^{-1}$. One caveat however is that stellar rotation is measured via its line broadening effects and thus is attenuated by a factor $\sin i$ (being the inclination angle). However, if $\sin i=0$, then the binary system is in the plane of the sky and should have a relatively high proper motion.

Marietta et al. (2000) performed detailed theoretical calculations exposing different donors to a supernova's ejecta and exploring the state of the donor post-explosion. The main-sequence and subgiant companions will keep most of their envelope and will be over-luminous for the next few millennia (confirmed by Pakmor et al. 2008). In stark contrast, the red giant companion will loose most of the loosely bound envelope and will appear as an under-luminous O- or B-star. In addition, their simulations show that all of the companions are unlikely to accrete much of the ejecta and thus probably won't show any abundance anomalies.

In summary, we expect a luminous surviving donor which might have a slightly unusual velocity and probably is highly rotating.

\section{The Hunting Grounds}

To investigate the nature of progenitors observationally, Ruiz-Lapuente (2004) have tried to directly detect donor stars in SNe Ia remnants within the Milky Way. They have identified two historical Galactic SNe Ia well suited to this task - SN 1006 and SN 1572 (Tycho's SN). Both remnants are young (1,000 and 440 years old, respectively), almost certainly SNe Ia based on their observational signatures (Badenes et al. 2006; Ruiz-Lapuente 2004; Krause et al. 2008; Rest et al. 2008), and are not overwhelmed by Galactic extinction. We have followed up Ruiz-Lapuente's (2004) work with Subaru and Keck high-resolution spectroscopy of stars in SN 1572. Furthermore we have scrutinized the central stars of SN 1006 with the FLAMES multi-object high-resolution spectrograph

\subsection{SN 1572}

There are six candidate stars close to the remnant's center of SN 1572 (see Figure 1). Ruiz-Lapuente et al. (2004) suggested the subgiant Tycho-G as a candidate due to its 


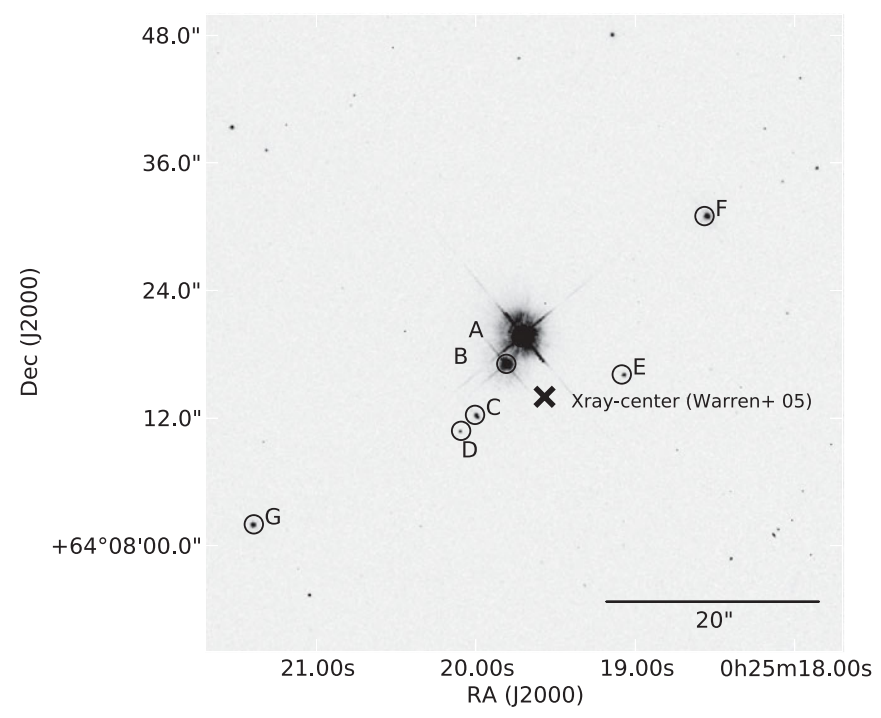

Figure 1. HST image of the central candidates of SN 1572 [HST data from Ruiz-Lapuente et al. (2004)]

unusual radial velocity, proper motion, and a distance compatible with the remnant. The main caveat of this result, however, was the seemingly large distance to the remnant's geometric center in the plane of the sky. A follow-up paper by González Hernández et al. (2009) revised the radial velocity of Ruiz-Lapuente et al. (2004), but confirmed the stellar parameters. In addition, González Hernández et al. (2009) measured an enhanced nickel abundance for Tycho-G, which is explained by the accretion of ejecta material onto the donor. Kerzendorf et al. (2009) confirmed the radial velocity and stellar parameters of González Hernández et al. (2009) by using Subaru HDS spectroscopy, but also found no rotation for Tycho-G (rotation less than the instrumental profile of $7.5 \mathrm{~km} \mathrm{~s}^{-1}$ ). Finally, they suggested that Tycho-G is an unrelated background interloper (conceding that there are a priori unlikely scenarios for Tycho-G as a donor).

This work has analyzed the same Keck HIRES spectra of Tycho-G (to be published in Kerzendorf et al. 2012b, in prep.). In our analysis of Tycho-G we confirm González Hernández et al. (2009)'s and Kerzendorf et al. (2009)'s stellar parameters, rotation and kinematic signature. In contrast to González Hernández et al. (2009), we find a nickel abundance consistent with $[\mathrm{Ni} / \mathrm{Fe}]=0$. In addition, we compare Tycho-G's anomalous proper motion with similar stars from the proper motion catalog PPMXL (Roeser et al. 2010) and find other stars with similar or higher proper motion. All this suggests that Tycho-G is an unrelated background star.

In addition, we analyzed Keck HIRES spectra of A, B, C, D and E. None of them show unusual kinematics, rotation, or state except for Tycho-B. Tycho-B is a relatively hot star with $T_{\text {eff }}=10000 \mathrm{~K}, \log g=4$, and an $[\mathrm{Fe} / \mathrm{H}] \approx-1$, with a very high rotation of $170 \mathrm{~km} \mathrm{~s}^{-1}$. These signs initially looked promising, but further analysis shows that Tycho-B has no unusually high velocity. As described in Section 2, rotation and escape velocity are linked such that a high rotational velocity requires a high escape velocity which is lacking for Tycho-B. Finally, we believe Tycho-B to be a foreground object, but our error range includes the distance of the remnant.

In summary, none of the candidate stars in SN 1572 are likely donors. One can make a case for either Tycho-G and Tycho-B; there are, however, serious caveats. 


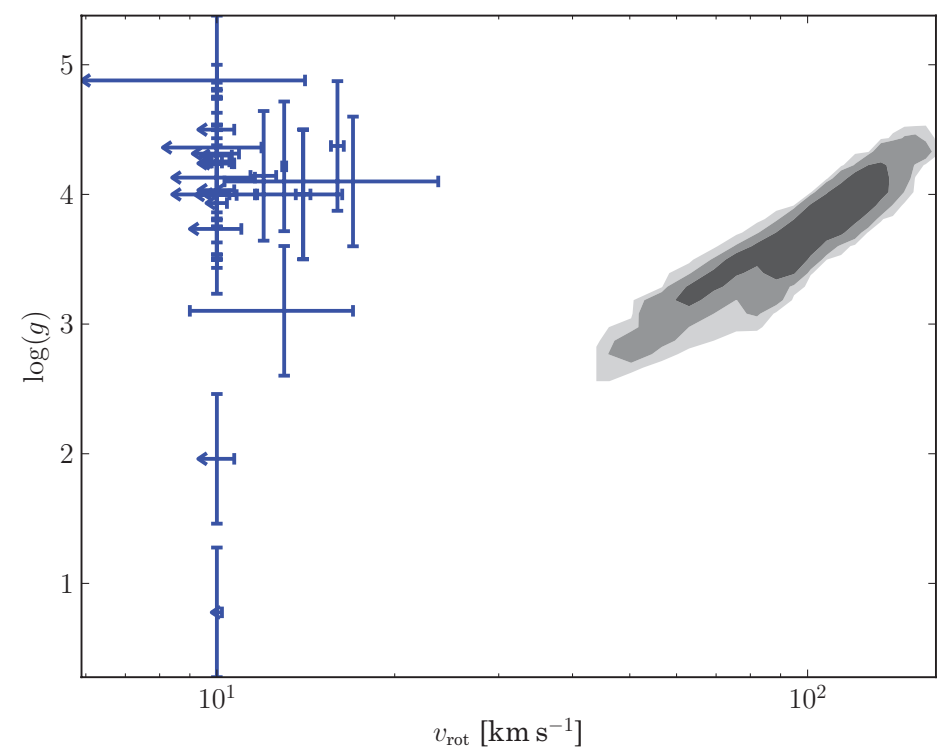

Figure 2. Comparison of the evolutionary state and rotational velocity of 55,000 binary synthesis single degenerate scenario progenitors (gray shades show $1-\sigma, 2-\sigma$ and $3-\sigma$ contours; data from Han 2008) with the measured rotation from this work. Due to the resolution of the spectrograph most of these stars only have an upper limit of the rotation speed of $v_{\text {rot }}=10 \mathrm{~km} \mathrm{~s}^{-1}$

\subsection{SN 1006}

The center of SN 1006 is much more crowded than that of SN 1572 and thus the observations are more challenging (to be published in Kerzendorf et al. 2012a, in prep.). This kind of work is uniquely suited to the multi-object high-resolution spectrograph FLAMES. For our candidates, we chose a search radius of $120^{\prime \prime}$, corresponding to the motion of a star traveling $1,250 \mathrm{~km} \mathrm{~s}^{-1}$ at $2.2 \mathrm{kpc}$ over 1,000 years. This generous choice, which is more than four times our maximum expected escape velocity suggested by Han (2008), was made to accommodate any errors in the choice of the center. Although the models predict the surviving companion to be several hundred $L_{\odot}$ (Marietta et al. 2000), we chose a limiting magnitude of $V=17.5\left[0.5 L_{\odot}(V)\right.$ at $2.2 \mathrm{kpc}$ including extinction of $\mathrm{E}(\mathrm{B}-\mathrm{V})=0.1]$ to accommodate a wide range of potential donor scenarios. An exposure time of 3.8 hours was chosen to obtain spectra with high enough quality to measure rotation and basic stellar parameters $(\mathrm{S} / \mathrm{N}$ ratio $>20)$. For completeness and to not waste fibers we chose additional stars down to a magnitude limit of $V=19$, which are only used for radial velocity measurements.

Our analysis show no unusual stars. The radial velocity measurements of all 78 stars are consistent with the kinematics from the Besançon Galactic dynamics model (Robin et al. 2003). In Figure 2 we show the rotational measurement and the surface gravity measurements of the bright sample $\left[L>0.5 L_{\odot}(V)\right.$ at $\left.2.2 \mathrm{kpc}\right]$ and compare them to the set of expected donor rotations by Han (2008). None of these stars show unusual rotation. In addition, no bright star shows sign of an unusual state.

The results in SN 1006 are consistent with the find in SN 1572 - no discernible donor.

\section{Conclusion}

The standard single degenerate scenario predicts a visible donor star post-explosion. In this work we have scrutinized the centers of two remnants (SN 1006 and SN 1572) 
and found no unambiguously identifiable donor star. There are some interesting options left in SN 1572 (see Tycho-G and Tycho-B), but they have strong caveats. A helium donor would certainly be too faint to be detected by our surveys, however these donors probably won't survive the explosion (priv. comm. Rüdiger Pakmor). Another option might be that the donor stars are not easily discernible from unrelated stars [in stark contrast to the theoretical results by Marietta et al. (2000) and Pakmor et al. (2008)]. Finally, the double-degenerate scenario is consistent with the results of both searches in SN 1572 and SN 1006.

As a next target we have chosen SN 1604. The morphology of this remnant is not as clean and spherical as SN 1006 and SN 1572, but SN 1604 still has a secure SNe Ia identity (Reynolds et al. 2007). A recent study by Chiotellis et al. (2011) suggests that a single degenerate scenario with an AGB star as a donor would explain all of the observed peculiarities and make the prediction that this star should be visible and very bright post-explosion.

We have obtained data with the WiFeS integral field spectrograph of SN 1604. The field of view for this instrument is rectangular with dimensions of $25^{\prime \prime} \times 38^{\prime \prime}$, sampled at $1.1^{\prime \prime}$. We have two overlapping fields that cover all stars at a projected velocity of $1,300 \mathrm{~km} \mathrm{~s}^{-1}$ (assuming a distance of $6 \mathrm{kpc}$ ). We are currently working on the data reduction and have no results as yet.

\section{References}

Badenes, C., Borkowski, K. J., Hughes, J. P., Hwang, U., \& Bravo, E. 2006, ApJ, 645, 1373, arXiv:astro-ph/0511140

Canal, R., Méndez, J., \& Ruiz-Lapuente, P. 2001, ApJ (Letters), 550, L53

Chiotellis, A., Schure, K. M., \& Vink, J. 2011, ArXiv e-prints, 1103.5487

González Hernández, J. I., Ruiz-Lapuente, P., Filippenko, A. V., Foley, R. J., Gal-Yam, A., \& Simon, J. D. 2009, ApJ, 691, 1, 0809.0601

Han, Z. 2008, ApJ (Letters), 677, L109, 0803.1986

Kerzendorf, W. E., Schmidt, B. P., Asplund, M., Nomoto, K., Podsiadlowski, P., Frebel, A., Fesen, R. A., \& Yong, D. 2009, ApJ, 701, 1665, 0906.0982

Kerzendorf, W. E., Schmidt, B. P., Laird, J. B., Carney, B. W., Podsiadlowski, P., \& Bessell, M. S. 2012a, in prep.

Kerzendorf, W. E., et al. 2012b, in prep.

Krause, O., Tanaka, M., Usuda, T., Hattori, T., Goto, M., Birkmann, S., \& Nomoto, K. 2008, Nature, 456, 617

Marietta, E., Burrows, A., \& Fryxell, B. 2000, ApJS, 128, 615

Pakmor, R., Röpke, F. K., Weiss, A., \& Hillebrandt, W. 2008, A\&GA, 489, 943, 0807.3331

Rest, A., et al. 2008, ApJ (Letters), 681, L81, 0805.4607

Reynolds, S. P., Borkowski, K. J., Hwang, U., Hughes, J. P., Badenes, C., Laming, J. M., \& Blondin, J. M. 2007, ApJ (Letters), 668, L135, 0708.3858

Robin, A. C., Reylé, C., Derrière, S., \& Picaud, S. 2003, A\&A, 409, 523

Roeser, S., Demleitner, M., \& Schilbach, E. 2010, AJ, 139, 2440, 1003.5852

Ruiz-Lapuente, P. 2004, ApJ, 612, 357

Ruiz-Lapuente, P., et al. 2004, Nature, 431, 1069 